\title{
sciendo
}

\section{Motor Control and Achilles Tendon Adaptation in Adolescence: Effects of Sport Participation and Maturity}

\author{
by \\ George Chalatzoglidis ${ }^{1}$, Fotini Arabatzi ${ }^{1}$, Evangelos A. Christou ${ }^{2,3}$
}

\begin{abstract}
An important but unresolved research question in adolescent children is the following: "Does sport participation interact with maturation to change motor control and the mechanical and morphological properties of tendons?" Here, we address this important research question with a longitudinal study around the age of peak height velocity (PHV). Our purpose was to characterize the interactive effects of maturation and sports participation on motor control and the mechanical and morphological properties of the Achilles tendon (AT) in adolescent athletes and nonathletes. Twenty-two adolescent athletes $(13.1 \pm 1.1$ years) and 19 adolescent non-athletes $(12.8 \pm 1.1$ years $)$ volunteered for this study. We quantified motor control as the coefficient of variation of torque during a ramp task. In addition, we quantified the AT morphological and mechanical properties using ultrasonography from 18 months before to 12 months after PHV. We found that motor control improved with maturation in both athletes and non-athletes. We found that athletes have a greater increase in body mass with maturation that relates to greater plantarflexion peak force and AT peak stress. Also, athletes have a thicker and longer AT, as assessed with resting cross-sectional area and length. Although the rate of increase in the morphological change with maturation was similar for athletes and nonathletes, the rate of increase in normalized AT stiffness was greater for athletes. This increased AT stiffness in athletes related to peak force and stress. In summary, maturation improves motor control in adolescent children. Further, we provide novel longitudinal evidence that sport participation interacts with maturation in adolescents to induce adaptive effects on the Achilles tendon morphology and mechanical properties. These findings have the potential to minimize the risk of injuries and maximize athletic development in talented adolescents.
\end{abstract}

Key words: peak height velocity, athletes, motor control, stiffness, stress, strain.

\section{Introduction}

An important time in human physical maturation is the age of the fastest growth in height, termed Peak Height Velocity (PHV). Although PHV varies among individuals, it is estimated to occur around the age of 12.1 years for girls and 13.7 years for boys (Granados et al., 2015). Body mass increases significantly around PHV (Malina, 2004, Malina and Bouchard, 1991), which results in rapid increases in muscle force and consequently a greater mechanical load on tendons (Lindgren, 1978, Lefevre et al., 1990). Further, there is significant evidence that our ability to control movements improves from childhood to early adulthood (Deutsch and Newell, 2001, Fox et al., 2014). Sport participation during adolescence also can induce tendon adaptations (O'Brien et al., 2010, Waugh et al., 2012, Magnusson et al., 2016), but the interaction of physical maturation and sports participation on motor control the tendon properties remains poorly understood. It is critical to understand this interaction to minimize the risk of injuries and maximize athletic development in talented adolescents. Here, we characterized the longitudinal changes in motor control and the properties of the Achilles tendon in adolescent

1 - Department of Physical Education and Sports Science at Serres, Aristotle University of Thessaloniki, Serres, Greece.

2 - Department of Applied Physiology and Kinesiology.

3 - Department of Neurology, University of Florida, Gainesville, FL, USA. 
athletes and non-athletes during a time period around PHV.

During adolescence, the time around $\mathrm{PHV}$ is critical for the development of the neuromuscular system. The immature neuromuscular system in adolescent children has been shown to be responsible for reductions in strength and power (Dotan et al., 2012). Children, also exhibit a reduced ability to control the motor output (Deutsch and Newell, 2001, Deutsch and Newell, 2002, Deutsch and Newell, 2006, Takahashi et al., 2003, Yan et al., 2000, Fox et al., 2014). For example, Deutsch and Newell (2001) have shown that children exhibit greater variability in force from 5-35\% MVC. Recent evidence from our lab expands these findings to showing that endpoint control is also impaired in children. However, the literature is limited in what happens to motor control around the age of PHV, when children go through the biggest mechanical transformation in life.

The mechanical properties of Achilles tendon change dramatically at the age of PHV. It is well known that body mass and muscle strength increase rapidly during the adolescent growth spurt, inducing a greater mechanical load on the Achilles tendon (Lindgren, 1978, Lefevre et al., 1990). Consequently, collagen fibril diameter, density and intra-fibrillary cross-linking can be affected and change material properties of the tendon (Radnor et al., 2018). These changes have been associated with increased stiffness $(k)$ and Young's modulus $(E)$ (Neugebauer and Hawkins, 2012, O'Brien et al., 2010, Waugh et al., 2012), whereas peak strain ( $\left.\varepsilon_{\text {peak }}\right)$ remains unchanged (Mogi et al., 2018). There is evidence, however, that $k$ or $E$ do not change significantly over a 6month period around the PHV (Neugebauer and Hawkins, 2012). Given that physical changes occur for about 6-7 years around PHV (Malina, 2004), the current knowledge about the effects of maturation on the mechanical properties of the Achilles tendon remains limited to very shorttime studies.

Sports participation and training also can influence the mechanical properties of the Achilles tendon in adolescence (Magnusson et al., 2016, Waugh et al., 2012, O'Brien et al., 2010). Although sports participation in children is more intense than in adults (PAC, 2020), its effect on the mechanical properties of the Achilles tendon during PHV remains unknown. Support to the idea that sports participation could interact with maturation and influence the mechanical and morphological (resting length, cross-sectional area) properties comes from studies on sports training. For example, in pre-pubertal children (Waugh et al., 2014) and adolescents (Mersmann et al., 2017b), the Achilles tendon stiffness increased significantly following 10 weeks of resistance training suggesting changes in underlying microstructure and collagen arrangement (Waugh et al., 2014). There is also evidence for Achilles and patellar tendon hypertrophy with participation in certain sports (Cassel et al., 2016). Recent findings, however, suggest that Achilles tendon stiffness was not different for preadolescent athletes and nonathletes following plyometric exercises (Pentidis et al., 2019). Thus, the effect of sports participation on the mechanical properties of the Achilles tendon and motor control at PHV remains unresolved.

The current literature on the interactive effect of maturation and sports participation, therefore, remains limited primarily to crosssectional studies. Here, we address this important research question with a longitudinal study around the age of PHV for adolescent athletes and non-athletes. Our purpose was to characterize the effects of maturation and sports participation on motor control and the mechanical and morphological properties of the Achilles tendon around the age of PHV. We tested the hypothesis that sport participation in adolescence will induce different adaptive patterns on the mechanical and morphological properties of the Achilles tendon with maturity and consequently motor control.

\section{Methods}

\section{Ethical approval.}

All students and parents provided informed consent. The Aristotle University of Thessaloniki Local Ethics Research Committee approved the study protocol and the research was conducted in accordance with the guidelines of the Declaration of Helsinki.

\section{Participants}

Fifty-seven pre pubertal children volunteered for the study (30 boys: $11.9 \pm 1.3$ years, $46.1 \pm 8.2 \mathrm{~kg}$, and $155.1 \pm 8.5 \mathrm{~cm} ; 27$ girls: $11.1 \pm 1.3$ years, $39.3 \pm 7.9 \mathrm{~kg}$, and $148.8 \pm 8.3 \mathrm{~cm}$ ). All 
participants were recruited from public schools in Greece and were healthy, without any disability or leg injury. Twenty-two athletes $(13.1 \pm 1.1$ years) and 19 non-athletes $(12.8 \pm 1.1$ years $)$ participated in the study. The athlete sample was derived from track and field participation (runners, sprinters, long jumpers), while the nonathlete sample derived from children that reported less than 3 hours of sport activity per week in school and no further sports training.

Experimental design

To estimate the age of PHV, we performed the same measurements under similar conditions for each participant every 6 months. The recommended time interval is $<1$ year (Mirwald et al., 2002), thus our measurements are within the suggested resolution. From the 57 adolescent participants, 41 of them (20 boys and 21 girls) were close to the age of PHV and were included in this study. Twenty-two of them were characterized as "adolescent athletes" $(13.1 \pm 1.1$ years) and 19 as "adolescent non-athletes" (12.8 \pm 1.1 years). Thus, within our sample, we have adolescents anywhere from 18 months before PHV to 12 months after PHV. Specifically, we classified the maturation progress into the following 6 stages: 18 months pre-PHV $(\mathrm{N}=24), 12$ months pre-PHV $(\mathrm{N}=33), 6$ months pre-PHV $(\mathrm{N}=41)$, PHV ( $\mathrm{N}=41), 6$ months post-PHV $(\mathrm{N}=17)$, and 12 months post-PHV $(\mathrm{N}=8)$. The number in parenthesis reflects the number of participants within each maturation stage.

\section{Measurements}

Anthropometric data

For each visit every six months, we recorded the participant's age, height, and weight. These measurements were necessary to estimate the PHV as recommended by (Mirwald et al., 2002). The age closer to the predicted age of PHV was defined as the age of peak height velocity (APHV) in each participant. In order to determine the reliability of this definition, the intraclass correlation coefficient (ICC) between the two ages (predicted and APHV) was calculated. The ICC rate for boys was 0.97, $(\mathrm{p}<.001)$ and for girls 0.77 , $(\mathrm{p}<.001)$. The acceptable limit of the coefficient $>0.7$ (Coffman et al., 2008), indicated that the predicted age of PHV was similar with APHV defined in the present study.

Plantarflexion contractions

To assess the strength of each participant at every 6-month visit, we asked the participants to perform ramp isometric plantarflexion contractions (IPF) in the prone position on a dynamometer bench (Cybex Humac Norm, CSMI, MA, USA; Figure 1A). The right foot was positioned perpendicular to the tibia (knee fully extended) and placed in the dynamometer's footplate with the right ankle in the neutral position (Muramatsu et al., 2001, Maganaris, 2002, Muraoka et al., 2004, Kubo et al., 2014). The foot was tightly secured with straps to the footplate and the hips were tightly strapped to the seat. The rotation axis of the ankle joint was carefully aligned to be parallel to the axis of the lever arm of the dynamometer and passing through the midpoint of the line connecting both malleoli (De Monte et al., 2006). Prior to testing, each participant performed a warming-up exercise (Maganaris and Paul, 1999, Rigby et al., 1959, Viidik et al., 1982, Schatzmann et al., 1998). Upon the instructor's request, participants performed two 10-s isometric plantar flexions increasing from $0 \%$ to $100 \%$ of the maximum torque at $20 \%$ of maximum torque every $2 \mathrm{~s}$ and holding maximally for $5 \mathrm{~s}$ (30-s inter-trial rest). Torque values were obtained from the best effort in which the isometric plantar flexion ramp was properly achieved and torque-time curves were linear in the ramp period without energy loss in the test. Motor control

Using the torque values from the isometric trial of plantar flexion ramp (see above) we quantified the force fluctuations around the force trajectory. We achieved this by removing the slow increase in force (tracking the ramp) by using a high-pass filter of the whole signal $(>0.1 \mathrm{~Hz})$. We then quantified the coefficient of variation $(\mathrm{CV})$ of force (standard deviation of force/mean force $\mathrm{x}$ 100). The CV of force is a more appropriate comparison between athletes and non-athletes and across maturity because different absolute force levels were exerted on tracking the ramp because of their strength.

Achilles tendon mechanical properties

To quantify the displacement of the distal myotendinous junction (MTJ) of the medial gastrocnemius (MG) during ramped IPF, we used a B-mode ultrasonography (SSD-3500, Aloka, Tokyo, Japan) with a $60-\mathrm{mm}$ electronic lineararray probe $(7.5 \mathrm{~Hz}$ wave frequency; Figure $1 \mathrm{~B})$. The probe was placed longitudinally at the 
position specified by a marker attached on the skin surface (Figure 1A). An echo-absorptive marker was attached to monitor possible motion of the probe on the skin during the measurement. Ultrasound images were digitally recorded at a sampling frequency of $25 \mathrm{~Hz}$. A Max TRAQ software (Max Traq Lite version 2.09, Innovision Systems, Inc. Columbiaville, Michigan. U.S.A) was used to digitize the spatial location of the MTJ in order to generate MTJ position-time data from the ultrasound video. AcqKnowledge Acquisition \& Analysis Software (BIOPAC Systems, Inc., USA) was used to synchronize the torque signal and ultrasound imaging data. Gastrocnemius myotendinous junction (MTJ) displacement was assumed to represent the change in the length of the AT (Maganaris and Paul, 1999, Muramatsu et al., 2001, Dick et al., 2016).

The effect of unavoidable joint angular rotation on the displacement of MTJ during the ramp contractions was taken into consideration by capturing (high-speed digital video, JVC 9800, frame rate $=120 \mathrm{~Hz}$ ) with a high-speed shutter to record plantar flexion motion (Magnusson et al., 2001b, Arampatzis et al., 2008). Five reflectors (2.5-mm radius) were placed on the $5^{\text {th }}$ metatarsal, lateral malleolus and lower extremity of the heel and two markers were placed on the foot plate (Figure 1A). Despite foot stabilization, displacement of the ankle joint was recorded during contraction, resulting in loss of alignment with the dynamometer rotation axis. To accurately calculate the angle of rotation in two dimensions, the position points of the reflectors were recorded during the ramp isometric plantar flexion and coordinate data from high-speed camera were down-sampled to match the ultrasound sampling frequency. The additional MTJ displacement was subtracted from recorded elongation $(\Delta \mathrm{L})$ to compensate for the influence of ankle joint rotation (Arampatzis et al., 2008, Magnusson et al., 2001a). The perpendicular distance from the center of rotation to the line of AT action was defined as AT moment arm (MA), which was calculated with the excursion method (Fath et al., 2010, Ito et al., 2000). Without changing the participant's position or the position of the ultrasound probe, passive AT behavior assessment was performed with the dynamometer rotating passively between $20^{\circ}$ dorsiflexion and $20^{\circ}$ plantarflexion at a speed of $5 \%$ s. The first derivative of the polynomial for angle $0^{\circ}$ was calculated to find the slope of the curve which represents the moment arm (Fath et al., 2010). Previous studies reported similar AT moment arm estimations of tendon excursion method between rest and MVC over any given foot rotation step (Maganaris, 2000, Maganaris et al., 2000). The tendon's force was calculated by the equation: $\mathrm{F}=M / d$ where $M$ is the plantarflexion moment and $d$ the AT MA length.

In this study, the force for isometric plantar flexion was calculated at angle $0^{\circ}$. The force resulting from this calculation represents the total force of all the ankle muscles transferred through the AT. Stiffness $(\mathrm{N} / \mathrm{mm})$ is defined as the slope of the linear portion of force $(\mathrm{F})$-elongation $(\Delta \mathrm{L})$ curve ranged from $60 \%$ to $90 \%$ of the maximum force were selected to be close to the linear portion of the force-elongation curve (Waugh et al., 2012). A 1st order polynomial was applied where the slope $\left(R^{2}=0.9\right)$ of the linear region of the curve $\mathrm{F}-\Delta \mathrm{L}$ was equal to the $\mathrm{k}$. Measurement of AT resting length (RL) was performed by sagittal imaging where the tendon boundaries were defined from AT insertion on the calcaneal tuberosity to the MTJ of gastrocnemius medialis (GM). To measure CSAAT, a transverse section was visualized using ultrasound (Maganaris, 2002). A special gel patch (gel pad Aquaflex, $2 \times 9 \mathrm{~cm}$ Parker Laboratories, Inc., Fairfield, NJ USA) was used to enhance acoustic conductivity and obtain clearer images where CSA AT $_{\text {in }}$ thinner and receives the greatest pressure (Voigt et al., 1995). The minimum CSAAT was the one accepted for the measurement. This ultrasonography technique is widely used a measurement of cross-sectional area of the AT (Waugh et al., 2012, Kubo et al., 2014, Mogi et al., 2018, Mogi, 2020). The reliability of measurements of CSAAT was investigated in a pilot study, 10 participants aged 9.5-14.5 performed the same test on 2 different days by two experienced observers. There were no significant differences between the test and retest

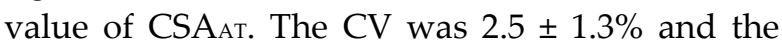
RMSE was, $2.2 \mathrm{~mm}^{2}$, a CV of $<5 \%$ is deemed acceptable (Campbell et al., 2007).

Stress $(\sigma)$, determined as the force per unit area, was calculated using the minimum recorded $\mathrm{CSA}_{\mathrm{AT}}$ from each participant according to the equation: $\sigma=F / A$ where $\mathrm{F}$ was the $\mathrm{AT}$ force $(\mathrm{N})$ 
and A was the minimum CSAAT $\left(\mathrm{mm}^{2}\right)$. Tendon strain $(\varepsilon)$ was defined as tendon's elongation normalized to its resting length according to the following equation: $\varepsilon=(\Delta \mathrm{L} / \mathrm{L} 0) \cdot 100 \%$ where $\mathrm{L}_{\mathrm{o}}$ and $\Delta \mathrm{L}$ represent the resting length and elongation of the Achilles tendon, respectively. The $E$ was calculated as the slope of the linear portion of stress $(\sigma)$ - strain $(\varepsilon)$ curve between $60 \%$ and $90 \%$ MVC and this was extrapolated to zero stress to yield the tendon resting length according to equation: $E=\mathrm{d} \sigma / \mathrm{d} \varepsilon$.

Analog signals from the dynamometer were amplified and synchronized with data from the ultrasound system using a DA $100 \mathrm{~B}$ amplifier (Biopac Systems. Inc., Goleta, CA, common mode rejection ratio $>90 \mathrm{db}$, bandwidth $=0.05-500 \mathrm{~Hz}$ ). Torque data were recorded by the dynamometer at a sampling frequency of $100 \mathrm{~Hz}$.

\section{Statistical analyses}

The statistical analysis was performed in SPSS (IBM Corp., Version 25.0, Armonk, New York, USA). Normal distribution of the data was tested using the Kolmogorov-Smirnov test. The independent variables for this study were the maturation (6 stages) and sport participation (athletes vs. non-athletes), whereas the dependent variables included: body mass, $\mathrm{CV}$ of torque, $\mathrm{AT}$ cross sectional area (CSAAT), AT resting length $(\mathrm{RL})$, peak force $\left(\mathrm{F}_{\text {peak }}\right)$, peak force normalized to body mass ( $\left.F_{\text {rel }}\right)$, stiffness $(k)$, stiffness normalized to $F_{\text {rel }}\left(k_{\mathrm{FM}}\right)$, Young's modulus $(E)$, Young's modulus normalized to $F_{\text {rel }}\left(E_{\mathrm{FM}}\right)$, peak strain $\left(\varepsilon_{\text {peak }}\right)$ and peak stress $\left(\sigma_{\text {peak }}\right)$.

We performed a repeated measure analysis of variance (RM ANOVA) to determine if sport participation interacted with maturity in the last 6 months (6 months pre-PHV and PHV) to affect the dependent variables. We used a linear mixed models (LMM) analysis to examine if athletes and non-athletes changed the dependent variables differently across the 6 stages of maturity. Additionally, LMM was used to compare CV of torque, $k, E$ and $\sigma_{\text {peak }}$ as function of $\mathrm{F}_{\text {peak, }}$ body mass and maturation as independent variables. The estimation method of -2 Restricted Log Likelihood (-2RLL) was used as the main criterion for comparison with other models and according to the best fit of the model. Additionally, -2RLL was more accurate than Full Maximum Likelihood as the number of participants $(\mathrm{N}=41)$ is small (Twisk, 2006, Singer, 2003). Pairwise comparisons between levels of selected main effects were conducted using Bonferroni confidence interval adjustment to determine the mean differences between athletes and nonathletes. Data are reported as mean \pm SD. Statistical significance was considered at a $p$ value $<0.05$.

\section{Results}

\section{Changes close to PHV}

We compared the body mass, CV of torque, and mechanical and morphological properties of the Achilles tendon for athletes and non-athletes at two maturity stages (6 months before PHV and $\mathrm{PHV})$. We chose this comparison because we had data from all participants at these two stages $(\mathrm{N}=41$; Table 1) and the only available longitudinal study compared adolescents 6 months apart around the age of PHV (Neugebauer and Hawkins, 2012).

The CV of torque was not significantly different for the two maturity stages or sport participation groups $(p>0.2)$. Similarly, the group $x$ sport participation interaction was not significant $(p>0.2)$. There was a significant main effect for maturation stage (6 months pre vs. PHV) showing that body mass, CSAAT, RL, Fpeak, Frel, E, and $\sigma_{\text {peak }}$ increased at the 6 months before PHV (all $\mathrm{p}<0.01$; Table 1). The main effect for sport participation (athletes vs. non-athletes) and the maturation stage $x$ sport participation interaction were not significant for any of the dependent variables ( $p>0.2)$. Thus, there is a change in body mass and various mechanical and morphological properties in the 6 months prior to PHV, but these changes are not significantly different for athletes and non-athletes.

\section{Longitudinal changes before and after PHV}

Given that we had a different number of observations for each maturation stage, we used linear mixed models (LMM) to examine the interactive effect of maturation and sport participation. We examined a maturation period of a 30-month span around PHV (pre 18 months, pre 12 months, pre 6 months, PHV, post 6 months, post 12 months).

CV of torque

The main effect for maturation (time) was significant $(p<0.01)$ indicating that the $\mathrm{CV}$ of torque decreased with maturation $(b=-1,24 \% \mathrm{CI}$ 2.36 to -0.13 , $\mathrm{p}=0.029$; Figure 2). The $\mathrm{CV}$ of torque 
was not significantly different for athletes and non-athletes $(b=0.48$ [-1.23-2.20], $p=0.577)$ and the interaction between maturation and sports participation was not significant, $(b=0.508$ [-1.102.12], $\mathrm{p}=0.535)$.

Body mass

The main effect for sport participation (athletes vs. non-athletes) and maturation (time) were significant $(\mathrm{p}<0.01)$ indicating that athletes had more body mass than non-athletes $(b=5$ [1.618.37], $\mathrm{p}=0.004$ ) and that body mass increased with maturation (Table 2). Most importantly, the interaction between maturation and sports participation was significant, indicating that body mass increased more in athletes than non-athletes $(b=2.06 \quad[0.04-4.07], \quad p=0.046 ;$ Figure 3A). Comparing the maturation slope for athletes and non-athletes for body mass in Figure 3A, it appears that the differences initiated 6 months before PHV. These differences continued to occur until 12 months post PHV because body mass kept increasing in athletes, whereas there was a plateau in non-athletes.

Peak force

The main effect for sport participation (athletes vs. non-athletes) and maturation (time) were significant $(p<0.01)$ indicating that athletes were stronger than non-athletes $(b=405.1$ [166.7643.5], $\mathrm{p}=0.001$ ) and that plantarflexion strength increased with maturation (Table 2; Figure 3B). The interaction between maturation and sports participation was not significant $(b=76.02[-58.82$ 210.60], $\mathrm{p}=0.264$ ). When we normalized peak force to body mass (Frel) we find a significant increase with maturation $(\mathrm{p}=0.046)$ but no difference between athletes and non-athletes $(b=2.70$ [-1.436.83], $\mathrm{p}=0.197)$.

Peak stress

The main effect for sport participation and maturation were significant $(\mathrm{p}<0.01)$ indicating that athletes exhibited increased peak stress compared with non-athletes $(b=5.02$ [0.78-9.24], $\mathrm{p}=0.021)$ and that peak stress increased with maturation ( $b=3.16$ [1.73 to 4.59], $\mathrm{p}<0.001$; Table 2). The interaction between maturation and sports participation was not significant $(b=0.29$ [-2.593.18], $\mathrm{p}=0.840$ ).

Associations

To determine the relation of body mass to peak force and peak force to peak stress in athletes and non-athletes we performed the following linear regressions: 1) Body mass and peak force - body mass associated significantly with peak force in both athletes $\left(\mathrm{R}^{2}=0.53\right.$; $\left.\mathrm{p}<0.01\right)$ and non-athletes $\left(R^{2}=0.30 ; p<0.01\right.$; Figure $\left.3 C\right)$. The slope was greater for athletes ( $47.1 \mathrm{vs.} 31.5 \mathrm{~N} / \mathrm{kg}$ ) suggesting that the maturation-dependent increases in muscle mass result in greater increases in peak force. 2) Peak force and peak stress - peak force associated significantly with peak stress in both athletes $\left(\mathrm{R}^{2}=0.30 ; \mathrm{p}<0.01\right)$ and non-athletes $\quad\left(R^{2}=0.60 ; \quad p<0.01 ; \quad\right.$ Figure $\left.3 D\right)$. However, the slope between the two variables was smaller $(0.01$ vs. $0.02 \mathrm{MPa} / \mathrm{kN})$ for athletes suggesting that the maturation-dependent increases in strength (muscle mass) result in lesser peak stress in the Achilles tendon. Thus, it appears that sport participation increases body mass at a greater rate in adolescent athletes than non-athletes, which leads to greater plantarflexion strength. However, the strength-induced increases in Achilles tendon peak stress is lesser in athletes than non-athletes, likely due to parallel changes in the internal structure of the tendon.

Morphological changes of the Achilles tendon

We quantified the Achilles tendon morphological changes with the resting crosssectional area (CSAAT) and length. The main effect for sport participation (athletes vs. non-athletes) and maturation (time) were significant $(p<0.01)$ indicating the following: Athletes had a greater $\mathrm{CSA}_{\text {AT }}(\mathrm{b}=0.027$ [0.016-0.038], $\mathrm{p}<0.001)$ and resting length $(b=0.62$ [0.19-1.04], $\mathrm{p}=0.005)$ than nonathletes (Figure 4; Table 2). Maturation increased the CSAAt $(b=0.013$ [0.01-0.02], $p<0.001$; Figure 4A) and resting length $(b=0.73$ [0.57-0.89], $p<0.001$; Figure 4B) of the Achilles tendon for both athletes and non-athletes. The interaction between sport participation $x$ maturation on CSAAT or resting length did not reach significance $(b=0.1[-0.2-1.1]$, $\mathrm{p}=0.06$ and $\mathrm{b}=0.09 \quad[-0.15-0.33], \quad \mathrm{p}=0.464$, respectively). Therefore, although athletes have a thicker and longer Achilles tendon, the rate of increase in these morphological changes appears to be similar for the two groups.

Structural changes of the Achilles tendon

We quantified the Achilles tendon structural changes independent of body weight and peak force with the normalized stiffness $\left(k_{\mathrm{FM}}\right)$ and Young's modulus $\left(E_{\mathrm{FM}}\right)$. Both of these measures were normalized to $F_{\text {rel }}$ (peak force normalized to body mass). There was a main effect for 
maturation for both measures of the Achilles tendon structure $(p<0.01)$. We found that both measures increased with maturation for athletes and non-athletes ( $k_{\mathrm{FM}}: \mathrm{b}=1.20$ [0.92-1.50], $\mathrm{p}<0.001$; $E_{\mathrm{FM}}: \mathrm{b}=4.25$ [3.09-5.42], $\left.\mathrm{p}=0.001\right)$. Furthermore, the interaction between sport participation and maturation was significant for the measures of structural change $\left(k_{\mathrm{FM}}: \mathrm{b}=0.45\right.$ [0.03-0.87], $\mathrm{p}=.038$; $E_{\text {Fм: }} b=1.80$ [0.07-3.52], $\left.p=.045\right)$. Specifically, the rate of increase in $k_{\mathrm{FM}}$ and $E_{\mathrm{FM}}$ was greater for athletes, suggesting that the rate of increase in normalized stiffness with maturation is greater for athletes than non-athletes (Figure 5).

Table 1.

Mean $( \pm \mathrm{SD})$ values 6 months before PHV and PHV (combined data). BM: body mass $(\mathrm{kg})$, $F_{\text {peak: }}$ peak force $(\mathrm{N}), F_{\text {rel: }}$ peak force normalized to body mass $(\mathrm{N} / \mathrm{kg}), R L$ : resting length, CSA Ат: minimum cross-sectional area $\left(\mathrm{mm}^{2}\right), k$ : stiffness $(\mathrm{N} / \mathrm{mm}), k_{\mathrm{FM}}$ : stiffness normalized to $F_{\text {rel }}(\mathrm{kg} / \mathrm{mm}), E$ : Young's modulus (MPa), EFM: Young's modulus normalized to $F_{\text {rel }}$ $\left(\mathrm{kg} / \mathrm{mm}^{2}\right), \varepsilon_{\text {peak: }}$ peak strain (\%), $\sigma_{\text {peak: }}$ peak stress $(\mathrm{MPa})$ of Achilles tendon. (combined data;

$\mathrm{N}=41$ )

\begin{tabular}{|c|c|c|}
\hline & $-6 \mathrm{mo}$ & PHV \\
\hline$B M$ & $47.3 \pm 9.5$ & $52.8 \pm 9.8$ \\
\hline$F_{\text {peak }}$ & $2108.5 \pm 501.8$ & $2586.7 \pm 696.5$ \\
\hline$F_{\text {rel }}$ & $45.2 \pm 9.4$ & $49.5 \pm 11.9$ \\
\hline$R L$ & $15.7 \pm 1.1$ & $17.2 \pm 1.3$ \\
\hline$C S A_{\mathrm{AT}}$ & $46.8 \pm 2.9$ & $49.3 \pm 3.3$ \\
\hline$k$ & $311.9 \pm 151.7$ & $372.2 \pm 153.9$ \\
\hline$k_{\mathrm{FM}}$ & $7.1 \pm 3.5$ & $7.8 \pm 3.3$ \\
\hline E & $1049.2 \pm 519.2$ & $1290.6 \pm 524.5$ \\
\hline$E_{\mathrm{FM}}$ & $23.8 \pm 12$ & $26.9 \pm 11.2$ \\
\hline$\varepsilon_{\text {peak }}$ & $6.8 \pm 1.1$ & $6.7 \pm 1$ \\
\hline$\sigma_{\text {peak }}$ & $40.9 \pm 14.8$ & $45.8 \pm 15.4$ \\
\hline
\end{tabular}


Table 2.

Mean $( \pm \mathrm{SD})$ values per stage of maturity around PHV for non-athletes $(\mathrm{NA} ; \mathrm{N}=19)$ and athletes $(\mathrm{A} ; \mathrm{N}=22)$. BM: body mass $(\mathrm{kg}), F_{\text {peak: }}$ peak force $(\mathrm{N}), F_{\text {rel: }}$ peak force normalized to body mass

$(\mathrm{N} / \mathrm{kg}), C S A$ ат: minimum cross-sectional area $\left(\mathrm{mm}^{2}\right), k$ : stiffness $(\mathrm{N} / \mathrm{mm}), k_{\mathrm{FM}}$ : stiffness normalized to $F_{\text {rel }}(\mathrm{kg} / \mathrm{mm}), E$ : Young's modulus (MPa), EFм: Young's modulus normalized to $F_{\text {rel }}\left(\mathrm{kg} / \mathrm{mm}^{2}\right), \varepsilon$ peak: peak strain (\%), $\sigma_{\text {peak: }}$ peak stress $(\mathrm{MPa})$ of Achilles tendon.

\begin{tabular}{|c|c|c|c|c|c|c|c|c|c|c|c|c|}
\hline \multirow[t]{2}{*}{ Stage } & \multicolumn{2}{|c|}{$-18 \mathrm{mo}(\mathrm{N}=24)$} & \multicolumn{2}{|c|}{$-12 \mathrm{mo}(\mathrm{N}=33)$} & \multicolumn{2}{|c|}{$-6 \mathrm{mo}(\mathrm{N}=41)$} & \multicolumn{2}{|c|}{ PHV $(\mathrm{N}=41)$} & \multicolumn{2}{|c|}{$+6 \mathrm{mo}(\mathrm{N}=17)$} & \multicolumn{2}{|c|}{$+12 \mathrm{mo}(\mathrm{N}=8)$} \\
\hline & NA & A & NA & A & NA & A & NA & $\mathrm{A}$ & NA & A & NA & $\mathrm{A}$ \\
\hline$B M$ & $\begin{array}{c}39.9 \pm \\
7\end{array}$ & $\begin{array}{c}41.2 \pm \\
11.4\end{array}$ & $\begin{array}{c}43.8 \pm \\
7.8\end{array}$ & $\begin{array}{c}44.2 \pm \\
10.9\end{array}$ & $\begin{array}{c}46.1 \pm \\
9\end{array}$ & $\begin{array}{c}48.3 \pm \\
10\end{array}$ & $\begin{array}{c}51.4 \pm \\
9.3\end{array}$ & $\begin{array}{l}54 \pm \\
10.2\end{array}$ & $\begin{array}{c}51.2 \pm \\
10.2\end{array}$ & $\begin{array}{c}58.3 \pm \\
8.2\end{array}$ & $\begin{array}{c}45.7 \pm \\
6.1\end{array}$ & $\begin{array}{c}61.9 \pm \\
7\end{array}$ \\
\hline$F_{\text {peak }}$ & $\begin{array}{r}1819.1 \\
\pm 512.7\end{array}$ & $\begin{array}{l}2089.1 \\
\pm 478.4\end{array}$ & $\begin{array}{r}1839.6 \\
\pm 412.5\end{array}$ & $\begin{array}{r}2130.3 \\
\pm 554.4\end{array}$ & $\begin{array}{c}1972.4 \\
\pm 422\end{array}$ & $\begin{array}{r}2225.9 \\
\pm 543.9\end{array}$ & $\begin{array}{l}2408.7 \\
\pm 625.9\end{array}$ & $\begin{array}{r}2740.4 \\
\pm 731.3\end{array}$ & $\begin{array}{l}2575.4 \\
\pm 477.1\end{array}$ & $\begin{array}{r}3143.8 \\
\pm 818.8\end{array}$ & $\begin{array}{c}2569.3 \\
\pm 397\end{array}$ & $\begin{array}{r}3286.4 \\
\pm 796.3\end{array}$ \\
\hline$F_{\text {rel }}$ & $\begin{array}{c}45.8 \pm \\
10.1\end{array}$ & $\begin{array}{c}51.6 \pm \\
7.2\end{array}$ & $\begin{array}{c}42.5 \pm \\
7.9\end{array}$ & $\begin{array}{c}48.8 \pm \\
9.4\end{array}$ & $\begin{array}{c}43.8 \pm \\
10.2\end{array}$ & $\begin{array}{c}46.4 \pm \\
8.6\end{array}$ & $\begin{array}{c}47.7 \pm \\
12.8\end{array}$ & $\begin{array}{l}51 \pm \\
11.1\end{array}$ & $\begin{array}{l}52 \pm \\
13.4\end{array}$ & $\begin{array}{c}53.6 \pm \\
10.8\end{array}$ & $\begin{array}{c}56.4 \pm \\
5.3\end{array}$ & $\begin{array}{c}53.3 \pm \\
13.5\end{array}$ \\
\hline$R L$ & $\begin{array}{c}14.5 \pm \\
1\end{array}$ & $\begin{array}{c}14.8 \pm \\
1\end{array}$ & $\begin{array}{c}14.9 \pm \\
1\end{array}$ & $\begin{array}{c}15.5 \pm \\
1.2\end{array}$ & $\begin{array}{c}15.4 \pm \\
0.9\end{array}$ & $\begin{array}{c}15.9 \pm \\
1.2\end{array}$ & $\begin{array}{c}16.9 \pm \\
1.2\end{array}$ & $\begin{array}{c}17.4 \pm \\
1.3\end{array}$ & $\begin{array}{c}17.1 \pm \\
0.7\end{array}$ & $\begin{array}{c}17.8 \pm \\
1.2\end{array}$ & $\begin{array}{c}16.9 \pm \\
0.6\end{array}$ & $\begin{array}{c}18 \pm \\
1.1\end{array}$ \\
\hline $\begin{array}{l}\text { CSA } \\
\text { AT }\end{array}$ & $\begin{array}{c}42.3 \pm \\
1.4\end{array}$ & $\begin{array}{c}46.7 \pm \\
3\end{array}$ & $\begin{array}{c}43.3 \pm \\
1.5\end{array}$ & $\begin{array}{c}47.6 \pm \\
2.6\end{array}$ & $\begin{array}{c}45.2 \pm \\
1.9\end{array}$ & $\begin{array}{c}48.1 \pm \\
2.9\end{array}$ & $\begin{array}{c}48.1 \pm \\
2\end{array}$ & $\begin{array}{c}50.4 \pm \\
3.8\end{array}$ & $\begin{array}{c}49.7 \pm \\
2.4\end{array}$ & $\begin{array}{c}52.4 \pm \\
3.4\end{array}$ & $\begin{array}{c}51 \pm \\
3.6\end{array}$ & $\begin{array}{c}52.8 \pm \\
3.1\end{array}$ \\
\hline$k$ & $\begin{array}{c}186 \pm \\
41.4\end{array}$ & $\begin{array}{c}191.4 \pm \\
34.2\end{array}$ & $\begin{array}{c}214.8 \pm \\
54.9\end{array}$ & $\begin{array}{c}206.7 \pm \\
60.4\end{array}$ & $\begin{array}{c}316.5 \pm \\
139.7\end{array}$ & $\begin{array}{c}307.9 \pm \\
164.6\end{array}$ & $\begin{array}{c}369 \pm \\
172\end{array}$ & $\begin{array}{c}375.1 \pm \\
140.4\end{array}$ & $\begin{array}{c}412 \pm \\
229\end{array}$ & $\begin{array}{c}485.2 \pm \\
212.2\end{array}$ & $\begin{array}{c}345 \pm \\
14.5\end{array}$ & $\begin{array}{c}492.7 \pm \\
116.8\end{array}$ \\
\hline$k_{\mathrm{FM}}$ & $\begin{array}{c}4.2 \pm \\
0.9\end{array}$ & $\begin{array}{c}3.8 \pm \\
0.9\end{array}$ & $\begin{array}{c}5.2 \pm \\
1.5\end{array}$ & $\begin{array}{c}4.4 \pm \\
1.6\end{array}$ & $\begin{array}{c}7.5 \pm \\
3.2\end{array}$ & $\begin{array}{c}6.7 \pm \\
3.8\end{array}$ & $8 \pm 3.5$ & $\begin{array}{c}7.6 \pm \\
3.2\end{array}$ & $\begin{array}{c}7.5 \pm \\
2.9\end{array}$ & $\begin{array}{c}9.1 \pm \\
3.4\end{array}$ & $\begin{array}{c}6.2 \pm \\
0.7\end{array}$ & $\begin{array}{c}9.3 \pm \\
0.9\end{array}$ \\
\hline E & $\begin{array}{c}639.9 \pm \\
159.8\end{array}$ & $\begin{array}{c}606.6 \pm \\
97.4\end{array}$ & $\begin{array}{c}736.8 \pm \\
182\end{array}$ & $\begin{array}{c}671.8 \pm \\
200.9\end{array}$ & $\begin{array}{r}1084.2 \\
\pm 497.2\end{array}$ & $\begin{array}{r}1018.9 \\
\pm 547.3\end{array}$ & $\begin{array}{c}1293 \pm \\
600.8\end{array}$ & $\begin{array}{c}1288.5 \\
\pm 463.4\end{array}$ & $\begin{array}{r}1393.3 \\
\pm 725.9\end{array}$ & $\begin{array}{r}1647.9 \\
\pm 731.2\end{array}$ & $\begin{array}{l}1142.7 \\
\pm 55.6\end{array}$ & $\begin{array}{r}1682.3 \\
\pm 432.3\end{array}$ \\
\hline$E_{\mathrm{FM}}$ & $\begin{array}{c}14.3 \pm \\
3.3\end{array}$ & $\begin{array}{c}12 \pm \\
2.7\end{array}$ & $\begin{array}{c}17.7 \pm \\
4.7\end{array}$ & $\begin{array}{c}14.3 \pm \\
5.1\end{array}$ & $\begin{array}{c}25.6 \pm \\
11.3\end{array}$ & $\begin{array}{c}22.3 \pm \\
12.6\end{array}$ & $\begin{array}{c}27.9 \pm \\
12.2\end{array}$ & $\begin{array}{c}26.1 \pm \\
10.4\end{array}$ & $\begin{array}{c}25.7 \pm \\
9.1\end{array}$ & $\begin{array}{c}30.8 \pm \\
11.4\end{array}$ & $\begin{array}{c}20.4 \pm \\
1.9\end{array}$ & $\begin{array}{c}31.5 \pm \\
1.9\end{array}$ \\
\hline$\varepsilon_{\text {peak }}$ & $\begin{array}{c}6.3 \pm \\
0.8\end{array}$ & $\begin{array}{c}6.8 \pm \\
0.9\end{array}$ & $\begin{array}{c}6.6 \pm \\
0.9\end{array}$ & $6.3 \pm 1$ & $7 \pm 1.2$ & $6.6 \pm 1$ & $\begin{array}{c}6.5 \pm \\
0.8\end{array}$ & $\begin{array}{c}6.8 \pm \\
1.1\end{array}$ & $\begin{array}{c}6.9 \pm \\
1.2\end{array}$ & $6.6 \pm 1$ & $7 \pm 1.2$ & $\begin{array}{c}6.8 \pm \\
0.8\end{array}$ \\
\hline$\sigma_{\text {peak }}$ & $\begin{array}{c}34.6 \pm \\
10.4\end{array}$ & $\begin{array}{c}38.2 \pm \\
12.6\end{array}$ & $\begin{array}{c}31.7 \pm \\
11.4\end{array}$ & $\begin{array}{c}40.6 \pm \\
16.7\end{array}$ & $\begin{array}{c}37.8 \pm \\
12.3\end{array}$ & $\begin{array}{c}43.6 \pm \\
16.5\end{array}$ & $\begin{array}{c}44.6 \pm \\
13.9\end{array}$ & $\begin{array}{c}46.9 \pm \\
16.8\end{array}$ & $\begin{array}{c}44.9 \pm \\
10.1\end{array}$ & $\begin{array}{l}51 \pm \\
13.5\end{array}$ & $\begin{array}{c}49.4 \pm \\
14.2\end{array}$ & $\begin{array}{c}51.7 \pm \\
15.3\end{array}$ \\
\hline
\end{tabular}



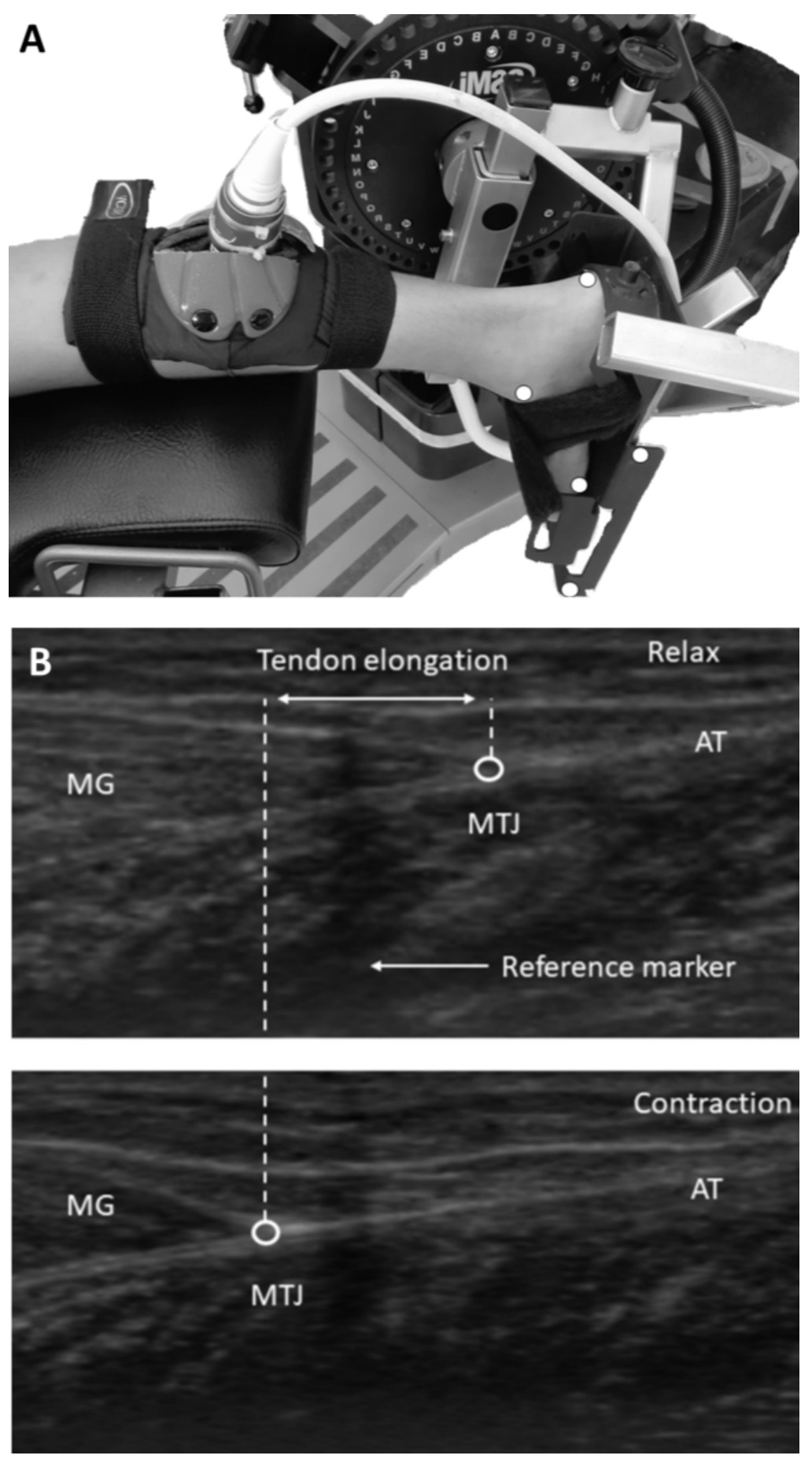

Figure 1

Experimental setup $(A)$ and ultrasound images of the Achilles tendon during the isometric plantarflexion (B). 


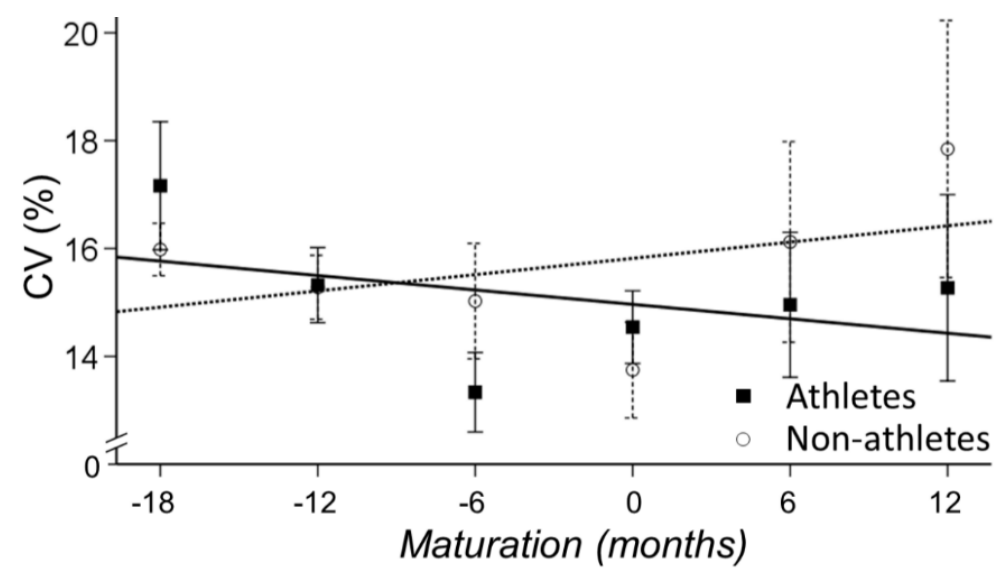

Figure 2

$C V$ of torque for athletes (solid line) and non-athletes (dashed line). Maturation changed the CV of torque similarly for the two groups.
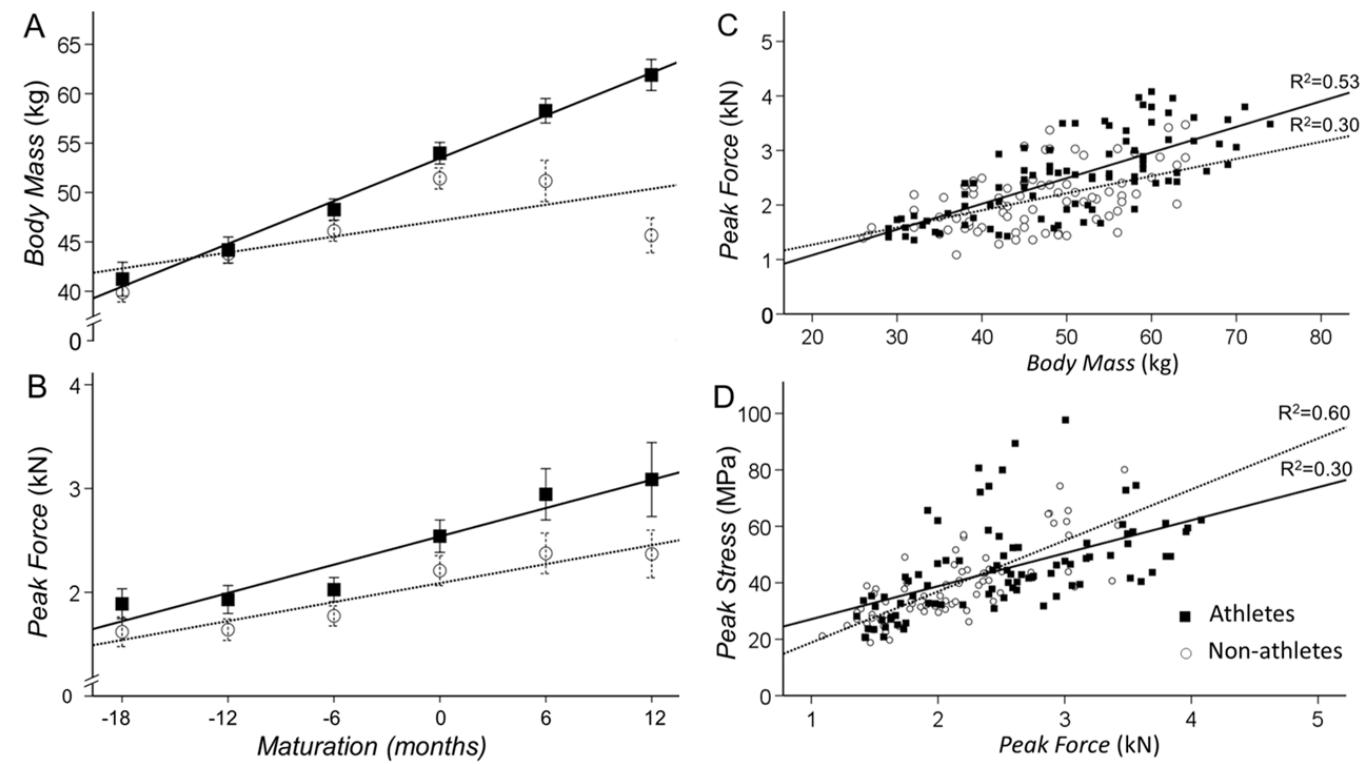

Figure 3.

Changes in body mass and peak force with maturity for athletes and non-athletes.

A. Body mass increased with maturity for both athletes (solid line) and non-athletes (dashed line). The increase in body mass with maturity was greater for athletes. B. Peak force was greater for athletes than non-athletes and increased with maturity for both groups. C. Peak force associated with body mass for both groups. The slope was slightly greater for athletes suggesting a greater increase in peak force with increased body mass than non-athletes. D. Peak stress associated with peak force for both groups. The slope was lower for athletes suggesting that the increase in peak force with maturation had a lesser influence on peak stress. 

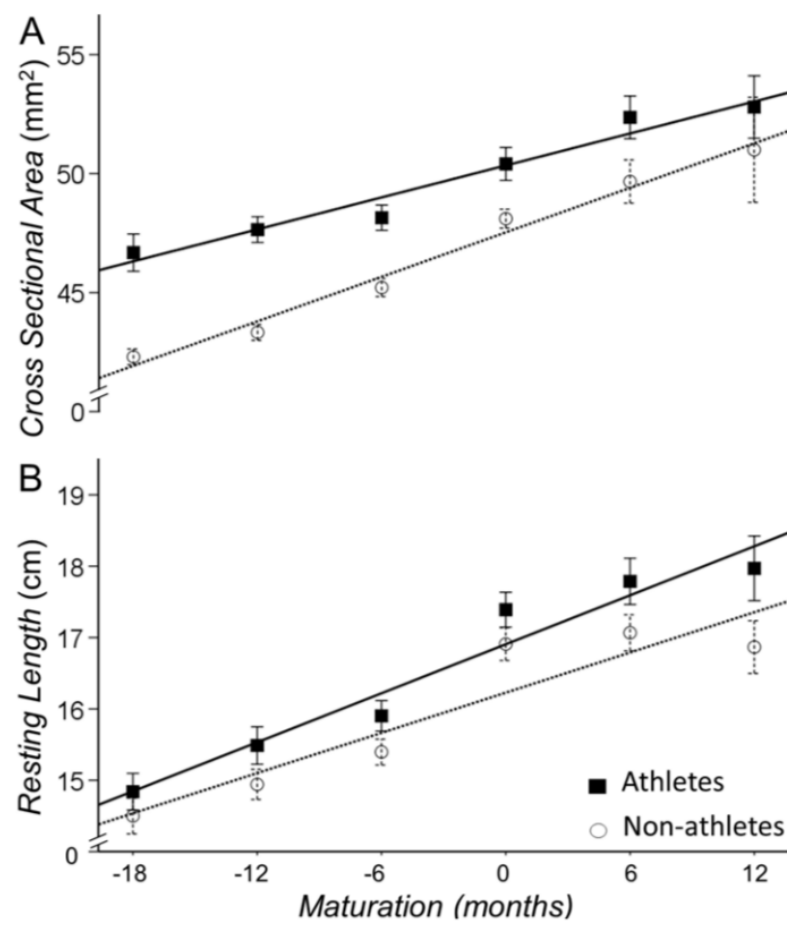

Figure 4

Cross sectional area and resting length of the Achilles tendon for athletes (solid line) and non-athletes (dashed line). Athletes had a greater cross-sectional area $(A)$ and resting length $(B)$ of the Achilles tendon than non-athletes. Maturation increased these morphological changes similarly for the two groups.

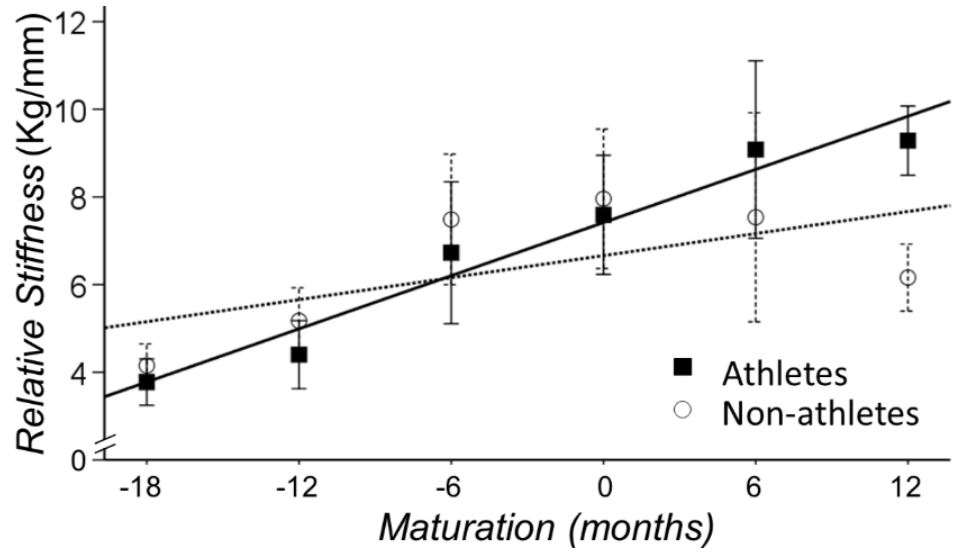

Figure 5

Relative stiffness in athletes and non-athletes. Relative stiffness increased at a greater rate with maturation in athletes (solid line) than non-athletes (dashed line). 


\section{Discussion}

Here, we examined if sport participation interacts with maturation during adolescence to change motor control and the mechanical and morphological properties of the Achilles tendon. To address this, we performed a longitudinal study around the age of peak height velocity (PHV) in adolescent athletes and non-athletes. We found that motor control improves with maturation but not with sport participation in adolescent children. Further, we found that sport participation clearly interacts with maturation and induces unique morphological and mechanical adaptations of the Achilles tendon. Specifically, athletes exhibited a greater increase in body mass and plantarflexion peak force with maturation, which related to increased Achilles tendon peak stress. Morphologically, the CSAAT was greater and AT length was longer in athletes, but the rate of increase with maturation was similar. Nonetheless, the rate of increase in the Achilles tendon stiffness was greater for athletes, as evidenced with normalized stiffness and Young Modulus. These findings provide original longitudinal evidence that sport participation alters the mechanical properties of the Achilles tendon but not necessarily the motor control of adolescent children.

Mechanical properties of the Achilles tendon

Our main interest was to determine if sport participation interacts with maturation to change motor control and the mechanical properties of the Achilles tendon. We found that motor control was influenced by maturation but not sport participation. We found that tendon stiffness and Young's modulus normalized to Frel (the ratio of peak force to body mass) increased at a greater rate with maturity for athletes than non-athletes. Thus, an important question related to this important finding is the following: "How does sport participation increase Achilles tendon stiffness at a greater rate with maturation?"

It is well accepted that body mass and force are loading factors on the Achilles tendon tissue, which can contribute to increased tendon stiffness (O'Brien et al., 2010). For example, Waugh et al. (2012) reported that peak strength and body mass have separate and additive effects on stiffness through development in children. Although, the effect depends on the timing and rate of maturation process (Malina et al., 2004, Malina and Bouchard, 1991, Waugh et al., 2012, Mogi et al., 2018), there is evidence that increased tendon stiffness in children occurs close to the PHV period (Radnor et al., 2018). Indeed, we found that the rate of increase with maturation in the normalized stiffness $\left(k_{\mathrm{FM}}\right)$ and Young modulus $\left(E_{\mathrm{FM}}\right)$ was greater for athletes, and this increased stiffness for athletes associated with greater peak stress but not with peak strain. Therefore, structural changes $\left(E_{\mathrm{FM}}\right)$ appear to be greater in athletes than non-athletes and these changes appear to be related to peak stress and force. This finding is in line with previous reports where trained pre-pubertal children had greater stiffness of AT than controls after 10 weeks of resistant training (Waugh et al., 2014) and late adolescent athletes had greater stiffness of patellar tendon than adolescents after 2 years training (Mersmann et al., 2017a). Maturity-dependent responses may be indicative of the coactive relationship between loading-specific adaptations and simultaneous growth and maturity-related changes (Lloyd et al., 2016).

The unique change of the Achilles tendon for athletes was not evident when we compared the mechanical properties 6 months before PHV with PHV. This finding suggests that a time period longer than 6 months before PHV is necessary to show the interaction between sport participation and maturation. This challenges previous studies that attempted to address this question by comparing mechanical properties in the 6-month period prior to PHV. Specifically, findings from our 6-month analysis are consistent with the observations of (Neugebauer and Hawkins, 2012), which showed that $k$ or $E$ do not change significantly over a 6-month period around the PHV. Regardless, our findings, which are based on longitudinal measurements, clearly suggest that sport participation interacts with maturation to increase not only the morphological properties of the Achilles tendon but also increases the structural strength of the Achilles tendon.

Morphological changes of the Achilles tendon

With maturation, common morphological tendon adjustments include an increase in crosssectional area and resting length. Our results show that the interaction between sport participation and maturation on the morphology of the Achilles tendon approached significance 
$(\mathrm{p}=0.06)$. Specifically, athletes had a greater AT cross-sectional area and resting length than nonathletes anywhere from 18 months before to 6 months after PHV (Figure 2B; Table 2). As Mogi (2019) has recently reported, the increase in cross sectional area just before the period of PHV is a protective mechanism to avoid possible injuries.

The effects of maturation on the morphological properties of tendons are conflicting. One study, found that the patellar tendon CSA increased $27 \%$ from mid to late adolescence (16-18 years) (Mersmann et al., 2017a), whereas others report reductions in tendon cross sectional areas in early adolescence (Neugebauer and Hawkins, 2012). These controversial reports about the tendon's hypertrophy in childhood to adulthood could be attributed to the age cohort and the inclusion of adolescents who participated in sports or not (Mersmann et al., 2017b, Cassel et al., 2017), and gender (Intziegianni et al., 2017). Our findings from this longitudinal study show that athletes had greater cross-sectional area and longer resting length of the Achilles tendon than non-athletes. Nonetheless, both athletes and non-athletes increase the cross-sectional area and resting length of the Achilles tendon with maturation at a similar rate. Thus, we show that both maturation and sport participation can increase the morphological properties of the Achilles tendon in adolescence.

The increase in tendon thickness and length with sport participation, at an earlier time from PHV may be an adaptive response to specific training-induced increase in muscle mass and strength (Falk and Eliakim, 2003, Waugh et al., 2014, Granacher et al., 2011). Our findings are consistent with Cassel et al. (2017) who reported greater tendon cross sectional area in athletes than non-athletes in specific sport activities. Nevertheless, our findings are in odds with other studies, which show that sports participation had no significant relationship with cross sectional area (Waugh et al., 2014, Neugebauer and Hawkins, 2012). One possible explanation is the type of sport training that adolescents participated in and thus a unique response to the variable mechanical loading (Radnor et al., 2018). Irrespective of the exact mechanism that induces Achilles tendon hypertrophy in athletes, this finding suggests that sport participation could be beneficial to adolescents.

What are the benefits of increased morphological and structural changes of the Achilles tendon with sport participation?

Our results clearly show that athletes have a greater CSAAт and stiffer Achilles tendon than non-athletes anywhere from 18 months before PHV to 6 months after PHV. Although the Achilles tendon stress was greater in athletes, the morphological adaptation of the tendon (i.e. greater cross-sectional area) reduces the tendon stress at a given tendon force. Therefore, these morphological and structural changes of the Achilles tendon with sport participation likely result in a reduced risk of injury. Such changes will also be protective against injuries that can occur during mid-adolescence when the changes in muscle strength and tendon loading capacity are not complimentary to each other (Mersmann et al., 2017a). Thus the mechanical loading that occurs with sport participation during adolescence can induce positive adaptations, which likely can reduce the risk for injuries while maximize athletic performance (Docking and Cook, 2019).

Limitations

In this study, we did not address the interaction of sex with sport participation and maturation. Female hormones in adolescence could result in differential changes in the crosssectional area and stiffness (Cassel et al., 2016) with maturity and sport participation. More research is needed to clarify the interaction between hormonal and mechanobiological processes (Mersmann et al., 2017a). In addition, the conclusions are drawn in the absence of examining neuromuscular changes with sport participation and maturity. Thus, future studies will benefit from the use of EMG to quantify the neuromuscular changes associated with sport participation and maturity. Finally, our findings are limited to adolescents and not adults. Future studies should determine if sport participation also influences adults.

\section{Conclusion}

Our longitudinal study shows that sport participation interacts with maturation during adolescence and induces unique morphological and mechanical adaptations of the Achilles tendon. Specifically, the Achilles tendon was thicker and longer in athletes, but most 
importantly the stiffness of the tendon increased at a greater rate in athletes than non-athletes. These findings provide original longitudinal evidence that sport participation alters the mechanical and morphological properties of the Achilles tendon in adolescence. These adaptive changes likely result in protection against possible injuries and improve tendon efficiency.

The authors have no conflicts of interest to disclose. The results of the study are presented clearly, honestly, and without fabrication, falsification, or inappropriate data manipulation.

\section{Acknowledgements}

Part of this work was presented in a poster presentation at the annual conference of the North American Society for the Psychology of Sport and Physical Activity (NASPSPA), June 13th-15th, 2013, at New Orleans, LA, USA (Abstract published in conference proceedings: Blischke, K., Malangré, A., Leinen, P. (2013). Sleeprelated offline-learning and task complexity in a motor skill requiring redundancy control. Journal of Sport and Exercise Psychology, 35, (Supplement), 21)

\section{References}

Arampatzis, a., monte, g. D. \& karamanidis, k. 2008. Effect of joint rotation correction when measuring elongation of the gastrocnemius medialis tendon and aponeurosis. J electromyogr kinesiol, 18, 503-8.

Campbell, m. J., machin, d. \& walters, s. J. 2007. Medical statistics : a textbook for the health sciences, chicester, england, john wiley \& sons, ltd.

Cassel, m., carlsohn, a., frohlich, k., john, m., riegels, n. \& mayer, f. 2016. Tendon adaptation to sport-specific loading in adolescent athletes. Int $j$ sports med, 37, 159-64.

Cassel, m., intziegianni, k., risch, 1., muller, s., engel, t. \& mayer, f. 2017. Physiological tendon thickness adaptation in adolescent elite athletes: a longitudinal study. Front physiol, 8, 795.

Coffman, d. L., maydeu-olivares, a. \& arnau, j. 2008. Asymptotic distribution free interval estimation for an intraclass correlation coefficient with applications to longitudinal data. Methodology-european journal of research methods for the behavioral and social sciences, 4, 4-9.

De monte, g., arampatzis, a., stogiannari, c. \& karamanidis, k. 2006. In vivo motion transmission in the inactive gastrocnemius medialis muscle-tendon unit during ankle and knee joint rotation. J electromyogr kinesiol, 16, 413-22.

Deutsch, k. M. \& newell, k. M. 2001. Age differences in noise and variability of isometric force production. J exp child psychol, 80, 392-408.

Deutsch, k. M. \& newell, k. M. 2002. Children's coordination of force output in a pinch grip task. Dev psychobiol, 41, 253-64.

Deutsch, k. M. \& newell, k. M. 2006. Age-related changes in the frequency profile of children's finger tremor. Neurosci lett, 404, 191-5.

Dick, t. J. M., arnold, a. S. \& wakeling, j. M. 2016. Quantifying achilles tendon force in vivo from ultrasound images. J biomech, 49, 3200-3207.

Docking, s. I. \& cook, j. 2019. How do tendons adapt? Going beyond tissue responses to understand positive adaptation and pathology development: a narrative review. J musculoskelet neuronal interact, 19, 300310.

Dotan, r., mitchell, c., cohen, r., klentrou, p., gabriel, d. \& falk, b. 2012. Child-adult differences in muscle activation--a review. Pediatr exerc sci, 24, 2-21.

Falk, b. \& eliakim, a. 2003. Resistance training, skeletal muscle and growth. Pediatr endocrinol rev, 1, 120-7.

Fath, f., blazevich, a. J., waugh, c. M., miller, s. C. \& korff, t. 2010. Direct comparison of in vivo achilles tendon moment arms obtained from ultrasound and mr scans. J appl physiol (1985), 109, 1644-52.

Fox, e. J., moon, h., kwon, m., chen, y. T. \& christou, e. A. 2014. Neuromuscular control of goal-directed ankle movements differs for healthy children and adults. Eur j appl physiol, 114, 1889-99.

Granacher, u., goesele, a., roggo, k., wischer, t., fischer, s., zuerny, c., gollhofer, a. \& kriemler, s. 2011. Effects and mechanisms of strength training in children. Int $j$ sports med, 32, 357-64. 
Granados, a., gebremariam, a. \& lee, j. M. 2015. Relationship between timing of peak height velocity and pubertal staging in boys and girls. J clin res pediatr endocrinol, 7, 235-7.

Intziegianni, k., cassel, m., hain, g. \& mayer, f. 2017. Gender differences of achilles tendon cross-sectional area during loading. Sports med int open, 1, e135-e140.

Ito, m., akima, h. \& fukunaga, t. 2000. In vivo moment arm determination using b-mode ultrasonography. Journal of biomechanics, 33, 215-218.

Kubo, k., teshima, t., hirose, n. \& tsunoda, n. 2014. A cross-sectional study of the plantar flexor muscle and tendon during growth. Int $j$ sports med, 35, 828-34.

Lefevre, j., beunen, g., steens, g., claessens, a. \& renson, r. 1990. Motor performance during adolescence and age thirty as related to age at peak height velocity. Ann hum biol, 17, 423-35.

Lindgren, g. 1978. Growth of schoolchildren with early, average and late ages of peak height velocity. Ann hum biol, 5, 253-67.

Lloyd, r. S., radnor, j. M., de ste croix, m. B., cronin, j. B. \& oliver, j. L. 2016. Changes in sprint and jump performances after traditional, plyometric, and combined resistance training in male youth pre- and post-peak height velocity. J strength cond res, 30, 1239-47.

Maganaris, c. N. 2000. In vivo measurement-based estimations of the moment arm in the human tibialis anterior muscle-tendon unit. J biomech, 33, 375-9.

Maganaris, c. N., baltzopoulos, v. \& sargeant, a. J. 2000. In vivo measurement-based estimations of the human achilles tendon moment arm. Eur j appl physiol, 83, 363-9.

Maganaris, c. N. \& paul, j. P. 1999. In vivo human tendon mechanical properties. J physiol, 521 pt 1, 307-13.

Maganaris, c. N., paul, j. P. 2002. Tensile properties of the in vivo human gastrocnemius tendon. J biomech, 35, 1639-46.

Magnusson, s. P., aagaard, p., dyhre-poulsen, p. \& kjaer, m. 2001a. Load-displacement properties of the human triceps surae aponeurosis in vivo. J physiol, 531, 277-88.

Magnusson, s. P., heinemeier, k. M. \& kjaer, m. 2016. Collagen homeostasis and metabolism. Adv exp med biol, 920, 11-25.

Magnusson, s. P., julsgaard, c., aagaard, p., zacharie, c., ullman, s., kobayasi, t. \& kjaer, m. 2001b. Viscoelastic properties and flexibility of the human muscle-tendon unit in benign joint hypermobility syndrome. Journal of rheumatology, 28, 2720-5.

Malina, r. M. \& bouchard, c. 1991. Growth, maturation, and physical activity, champaign, ill., human kinetics books.

Malina, r. M., bouchard, c., bar-or, o. 2004. Growth, maturation, and physical activity, champaign, human kinetics.

Malina, r. M., boushard, c. \& bar-or, o. 2004. Growth, maturation, and physical activity, champain, il usa, human kinetics.

Mersmann, f., bohm, s., schroll, a., boeth, h., duda, g. N. \& arampatzis, a. 2017a. Muscle and tendon adaptation in adolescent athletes: a longitudinal study. Scand j med sci sports, 27, 75-82.

Mersmann, f., charcharis, g., bohm, s. \& arampatzis, a. 2017b. Muscle and tendon adaptation in adolescence: elite volleyball athletes compared to untrained boys and girls. Front physiol, 8, 417.

Mirwald, r. L., baxter-jones, a. D. G., bailey, d. A. \& beunen, g. P. 2002. An assessment of maturity from anthropometric measurements. Medicine and science in sports and exercise, 34, 689-694.

Mogi, y. 2019. The relationship between the length and cross-sectional area of the achilles tendon and age at peak height velocity. Japan journal of human growth and development research, 2019, 23-31.

Mogi, y. 2020. The effects of growth on structural properties of the achilles and patellar tendons: a crosssectional study. Physiological reports, 8, e14544-e14544.

Mogi, y., torii, s., kawakami, y. \& yanai, t. 2018. A cross-sectional study on the mechanical properties of the achilles tendon with growth. Eur j appl physiol, 118, 185-194.

Muramatsu, t., muraoka, t., takeshita, d., kawakami, y., hirano, y. \& fukunaga, t. 2001. Mechanical properties of tendon and aponeurosis of human gastrocnemius muscle in vivo. J appl physiol (1985), 90, 1671-8.

Muraoka, t., muramatsu, t., fukunaga, t. \& kanehisa, h. 2004. Geometric and elastic properties of in vivo human achilles tendon in young adults. Cells tissues organs, 178, 197-203. 
Neugebauer, j. M. \& hawkins, d. A. 2012. Identifying factors related to achilles tendon stress, strain, and stiffness before and after 6 months of growth in youth 10-14 years of age. J biomech, 45, 2457-61.

O'brien, t. D., reeves, n. D., baltzopoulos, v., jones, d. A. \& maganaris, c. N. 2010. Mechanical properties of the patellar tendon in adults and children. J biomech, 43, 1190-5.

Pac 2020. Physical activity counsil's overview report on u.s. participation. Retrieved from http://www.physicalactivitycouncil.com/. 17/8/2020.

Pentidis, n., mersmann, f., bohm, s., giannakou, e., aggelousis, n. \& arampatzis, a. 2019. Triceps surae muscle-tendon unit properties in preadolescent children: a comparison of artistic gymnastic athletes and non-athletes. Front physiol, 10, 615.

Radnor, j. M., oliver, j. L., waugh, c. M., myer, g. D., moore, i. S. \& lloyd, r. S. 2018. The influence of growth and maturation on stretch-shortening cycle function in youth. Sports med, 48, 57-71.

Rigby, b. J., hirai, n., spikes, j. D. \& eyring, h. 1959. The mechanical properties of rat tail tendon. Journal of general physiology, 43, 265-283.

Schatzmann, 1., brunner, p. \& staubli, h. U. 1998. Effect of cyclic preconditioning on the tensile properties of human quadriceps tendons and patellar ligaments. Knee surg sports traumatol arthrosc, 6 suppl 1, s5661.

Singer, j. D., willett, j. B. 2003. Applied longitudinal data analysis: modeling change and event occurrence, oxford university press inc, usa.

Takahashi, c. D., nemet, d., rose-gottron, c. M., larson, j. K., cooper, d. M. \& reinkensmeyer, d. J. 2003. Neuromotor noise limits motor performance, but not motor adaptation, in children. J neurophysiol, 90, 703-11.

Twisk, j. W. R. 2006. Applied multilevel analysis, cambridge university press.

Viidik, a., danielsen, c. C. \& oxlund, h. 1982. On fundamental and phenomenological models, structure and mechanical-properties of collagen, elastin and glycosaminoglycan complexes. Biorheology, 19, 437451.

Voigt, m., bojsen-moller, f., simonsen, e. B. \& dyhre-poulsen, p. 1995. The influence of tendon youngs modulus, dimensions and instantaneous moment arms on the efficiency of human movement. $J$ biomech, 28, 281-91.

Waugh, c. M., blazevich, a. J., fath, f. \& korff, t. 2012. Age-related changes in mechanical properties of the achilles tendon. J anat, 220, 144-55.

Waugh, c. M., korff, t., fath, f. \& blazevich, a. J. 2014. Effects of resistance training on tendon mechanical properties and rapid force production in prepubertal children. J appl physiol (1985), 117, 257-66.

Yan, j. H., thomas, j. R., stelmach, g. E. \& thomas, k. T. 2000. Developmental features of rapid aiming arm movements across the lifespan. J mot behav, 32, 121-40.

\section{Corresponding authors:}

\section{Georgios Chalatzoglidis}

Laboratory of Neuromechanics, Department of Physical Education and Sport Science, Aristotle University of Thessaloniki, Serres, Agios Ioannis, 62110, Greece. Tel: +30 2321037622, +306977620694,

E-mail: gchalatzo@phed-sr.auth.gr,

Fotini Arabatzi

Laboratory of Neuromechanics, Department of Physical Education and Sport Science,

Aristotle University of Thessaloniki, Serres, Agios Ioannis, 62110, Greece.

E-mail: farabaji@phed-sr.auth.gr,

Evangelos A. Christou

Department of Applied Physiology and Kinesiology, ${ }^{3}$ Department of Neurology, University of Florida, Gainesville, FL, USA

E-mail: eachristou@ufl.edu, 